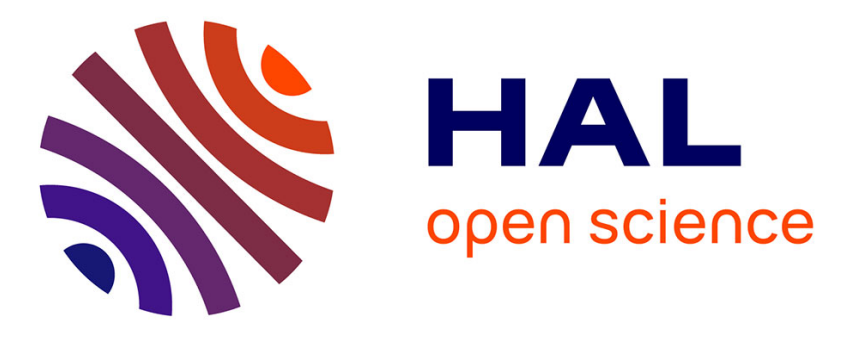

\title{
Redesign of a High-Pressure Compressor Blade Accounting for Nonlinear Structural Interactions
}

Alain Batailly, Mathias Legrand, Antoine Millecamps, Sébastien Cochon, François Garcin

\section{- To cite this version:}

Alain Batailly, Mathias Legrand, Antoine Millecamps, Sébastien Cochon, François Garcin. Redesign of a High-Pressure Compressor Blade Accounting for Nonlinear Structural Interactions. Journal of Engineering for Gas Turbines and Power, 2014, 137 (2), pp.022502. 10.1115/1.4028263 . hal-01120158

\author{
HAL Id: hal-01120158 \\ https://hal.science/hal-01120158
}

Submitted on 24 Feb 2015

HAL is a multi-disciplinary open access archive for the deposit and dissemination of scientific research documents, whether they are published or not. The documents may come from teaching and research institutions in France or abroad, or from public or private research centers.
L'archive ouverte pluridisciplinaire HAL, est destinée au dépôt et à la diffusion de documents scientifiques de niveau recherche, publiés ou non, émanant des établissements d'enseignement et de recherche français ou étrangers, des laboratoires publics ou privés. 


\title{
Redesign of a high-pressure compressor blade accounting for nonlinear structural interactions
}

\author{
Alain Batailly ${ }^{1, *}$, Mathias Legrand ${ }^{1}$, Antoine Millecamps ${ }^{2}$, Sébastien Cochon ${ }^{2}$, François Garcin ${ }^{2}$
}

\begin{abstract}
Recent numerical developments dedicated to the simulation of rotor/stator interaction involving direct structural contacts have been integrated within the Snecma industrial environment. This paper presents the first attempt to benefit from these developments and account for structural blade/casing contacts at the design stage of a high-pressure compressor blade. The blade of interest underwent structural divergence after blade/abradable coating contact occurrences on a rig test. The design improvements were carried out in several steps with significant modifications of the blade stacking law while maintaining aerodynamic performance of the original blade design. After a brief presentation of the proposed design strategy, basic concepts associated with the design variations are recalled. The iterated profiles are then numerically investigated and compared with respect to key structural criteria such as: (1) their mass, (2) the residual stresses stemming from centrifugal stiffening, (3) the vibratory level under aerodynamic forced response and (4) the vibratory levels when unilateral contact occurs. Significant improvements of the final blade design are found: the need for an early integration of nonlinear structural interactions criteria in the design stage of modern aircraft engines components is highlighted.
\end{abstract}

\section{Keywords}

Nonlinear dynamics, contact mechanics, aeronautical engine, rotor/stator interactions, vibrations.

\section{Nouvelle conception d'une aube de compresseur haute-pression en prenant en compte des interactions structurelles non-linéaires}

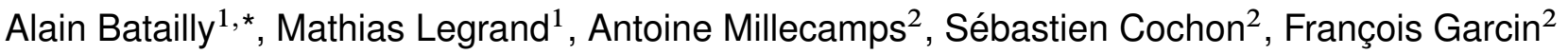

\begin{abstract}
Résumé
De récents travaux dédiés à la simulation du contact aube/carter en configuration non-accidentelle ont été intégrés dans l'environnement industriel de l'entreprise Snecma. Cet article présente la première tentative d'application de ces développements numériques pour prendre en compte des contacts structurels dans le processus de conception d'une aube de compresseur haute-pression. Un comportement vibratoire divergent a été mis en évidence pour l'aube étudiée à la suite de contacts aube/carter simulés sur un banc d'essai. Les améliorations de conception ont été faites en plusieurs étapes en modifiant significativement la loi d'empilage du profil d'aube tout en maintenant des performances aérodynamiques inchangées. Dans une première partie, la stratégie de conception est brièvement détaillée puis les concepts clés associés à l'évolution du profil d'aube sont détaillés. Les profils itérés font l'objet de plusieurs types de simulations numériques et les résultats obtenus sont confrontés à ceux du profil initial en se focalisant sur: (1) la masse, (2) les contraintes résiduelles associées au raidissement centrifuge, (3) le niveau de la réponse vibratoire du profil sous chargement aérodynamique et (4) les amplitudes vibratoires prédites lorsque des contacts aube/carter se produisent. Des améliorations nettes sont observées pour le profil optimisé et cet article souligne le besoin pour une intégration de critères de conception associées aux interactions structurelles non-linéaires dès la phase de conception des aubes.
\end{abstract}

Mots-clés

Dynamique nonlinéaire, mécanique numérique du contact, moteur aéronautique, interaction rotor/stator, vibrations.

1 - Laboratoire de Dynamique des Structures et Vibrations, Université McGill, 817 Sherbrooke Ouest, Pavillon McConnell, Salle 122, H3A-0C3, Montréal, Québec, Canada

2 - Snecma, site de Villaroche, Moissy-Cramayel, 77550, France

Auteur à contacter : Tél. : +15143985321; fax : +15143987365. Courriel : alain.batailly@mcgill.ca

\section{Introduction}

The existence of several nonlinear interfaces within aircraft engines and turbines in general—-such as the blade-tip/casing or the shaft/bearing interfaces-are propitious to the occurence of a large variety of rotor/stator interactions that may threaten the engine structural integrity. As a rule, modern designs feature tighter operating clearances in order to increase the engine efficiency. Consequently, structural contacts are now accepted as normal operating conditions and subsequent interactions such as the rub- bing of a single blade on the abradable coating [1, 2], modal interaction [3, 4] or backward and forward whirl motions [5], have been widely investigated over the last years. The growing understanding of these interactions allow designers to catch sight of robust structures with respect to structural contacts, in particular blades in order to mitigate events such as blade failure or blade loss. A numerical strategy dedicated to the analysis of blade/casing and blade/abradable coating interactions previously introduced $[6,7]$ has been implemented in the industrial envi- 
ronment of Snecma. This paper focuses on the first attempt to benefit from this strategy as early as the blade design stage.

The blade of interest belongs to the high-pressure compressor of a modern aircraft engine. At this stage of the engine, an abradable coating is deposited along the casing circumference in order to avoid damages when contacts occur. However, a structural divergence, defined as an abnormal increase of the amplitude of vibration of the blade possibly leading to blade failure for a given rotational frequency, was witnessed on a rig test after abradable coating/blade contacts. Such phenomenon was previously witnessed within a low-pressure compressor [8] and accurately predicted with the employed numerical strategy [7]. The interaction may be described as follows: blade/abradable coating contact is initiated due to the blade-tip displacement stemming from centrifugal effects. If the blade first eigenfrequency (some interactions have also been witnessed for the second eigenfrequency) is a multiple of the rotational frequency then the blade may impact the casing at the same location at every revolution. The abradable coating wear may then be localized. Thus, at every revolution, the blade/abradable coating clearance opens allowing for larger amplitudes of vibration of the blade. The blade amplitude of vibrations grows over time as the abradable coating is progressively removed until blade failure is witnessed [8].

The numerical analysis of such interaction is twofold: (1) explicit time integration [7] is first required in order to obtain quantitative results such as the abradable coating removal rate. It provides useful information regarding the blade vibratory response before a steady state is reached; and (2) more qualitative approaches [9] that bring meaningful results regarding the nature of the interaction assuming a steady state can be reached. While more costful in terms of computation time, the first type of strategy is the one that compares the best with experimental observations so far which explains why it is considered in this paper.

The first section focuses on typical blade design parameters. The notion of structural divergence is then recalled as the interaction scenario used for the definition of optimization criteria is presented. The three blade profiles obtained from the design process are then compared in terms of fundamental mechanical properties and vibratory behaviour for two given contact configurations with the casing. The last section of the paper provides additional insight on the key differences between the blade profiles using spectrograms.

There is no mention of aerodynamic performances in this paper since its focus is essentially structural. However, it has been checked that all the presented profiles feature identical aerodynamic performances through multistage 3D Navier-Stokes conputations of the high-pressure compressor focusing on the flow rate, the efficiency as well as the pressure ratio. Also, for the sake of confidentiality, all displacements, frequencies and rotational frequencies are normalized.

\section{Key parameters of a blade}

The blade of interest is pictured in Fig. 1. The associated finite element model contains 13,803 nodes and 7,388 tetrahedron quadratic finite elements. A blade is essentially an airfoil that extends radially between the blade root and the blade tip. Its profile consists of a series of basic profiles stacked on one another along a line connecting each profile centre of gravity [10]; this line is called the stacking law and is depicted as ((-- - )) in Fig. 1.

The stacking law is the fundamental design parameter of the blade. In our study, it is defined through a large number of parameters including the coordinates of the centre of gravity of each basic profile. The blade profiles presented in the following essentially feature distinct stacking laws. The modification of the stacking law has a direct impact on the blade vibratory behaviour through, for instance, a very strong influence over the stress field due to centrifugal stiffening within the blade.

From a structural standpoint, blade fundamental properties lie in (1) its mass, (2) the maximum value of centrifugal stresses, (3) the amplitude of its forced response to aerodynamic excitations. Based on the numerical strategy employed for the simulation of rotor/stator interactions, another property of the blade is of particular interest in this paper: the amplitude of its vibratory response for a given contact scenario over a properly selected rotational frequency range. Looking at the evolution of this amplitude over the rotational frequency range allows for the detection of structural divergences.

\section{Structural divergence and blade design}

As mentioned in the introduction, a structural divergence characterizes a rotational frequency for which the blade amplitude of vibration increases over time after contact is initiated with the abradable coating. Typically, a structural divergence leads to blade failure — as witnessed in [8] — and thus must be avoided. In order to predict structural divergences and the associated rotational frequencies, namely the critical rotational frequencies, the numerical strategy presented in [6] is used considering a deformed casing, assumed rigid enough not to vibrate when blade/abradable coating contacts occur[8, 2]. Actual casings within aircraft engines are never perfectly circular, it has been observed that their deformations are mostly along a two- or fournodal diameter free vibration mode thus leading to two or four privileged contact areas as represented in Fig. 2.

Accordingly, these two types of deformations are considered in the numerical simulations carried out in the following. The interaction scenario may be described as follows:

- Contact initiation: over the first revolutions of the blade, both the casing deformation and the blade-tip displacement due to centrifugal stiffening are progressively accounted for so that there is no blade/abradable coating penetration at $t=0 \mathrm{~s}$;

- Abradable coating removal: once contact occurs between the blade-tip and the coating, the blade starts vibrating and the abradable coating may be locally removed if the abradable elements plastify;

- Steady state: after several revolution with the blade impacting the abradable coating, several scenarios are possible: (1) contact is lost and the blade amplitude of vibrations vanish due to structural damping, (2) contact is maintained over time between the blade and the abradable coating which undergoes elastic deformations. A third scenario featuring a growing amplitude of vibration, as witnessed in [7], may also be considered.

For each rotational frequency, the blade makes 50 revolutions. It is observed that this number of revolutions is sufficient to reach steady state. Once steady state is reached, the focus is made on the amplitude of vibration of the blade plotted over the rotational frequency range considered. The calibration of the other simulation parameters such as the mechanical properties of the abradable coating and the initial blade-tip/casing clearance configurationthat are not mentioned here for the sake of confidentiality-has 

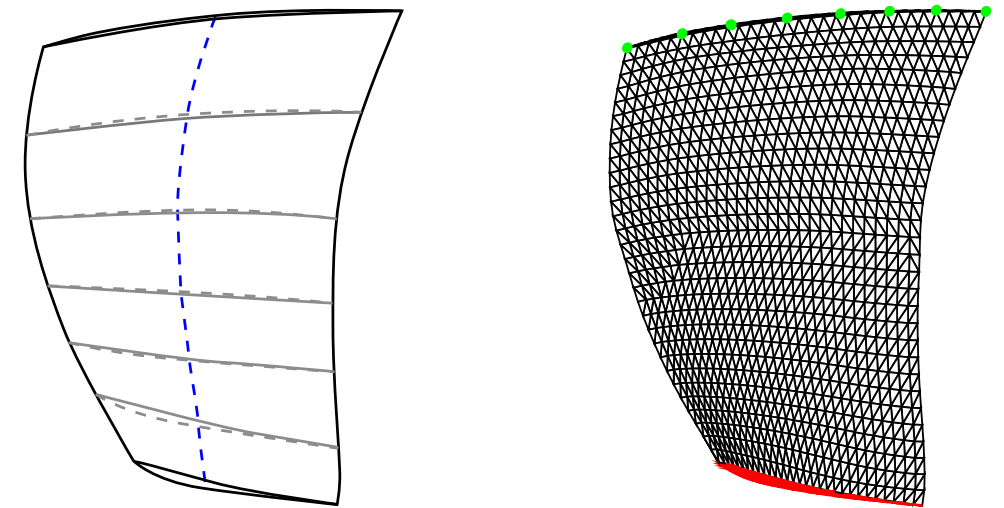

Figure 1. Stacking law and mesh (clamped area in red, contact nodes in green) of the blade of interest
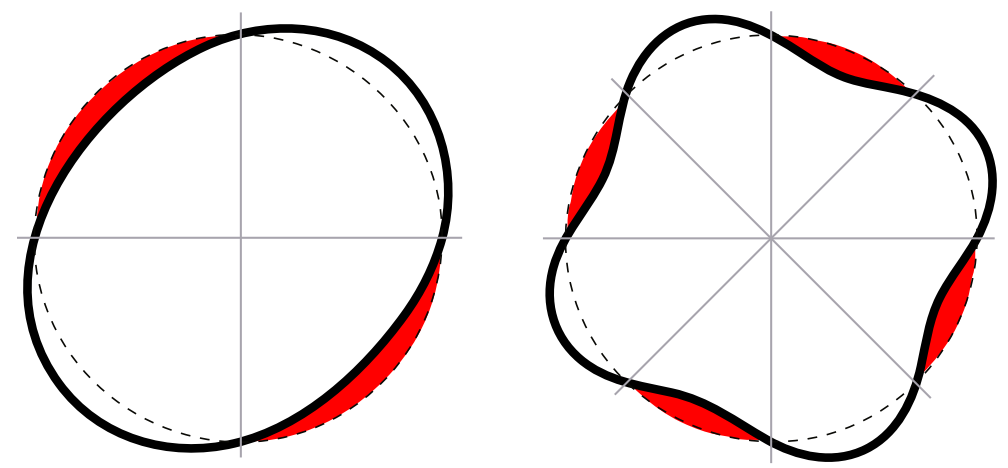

Figure 2. 2D planar representation of typical casing deformations

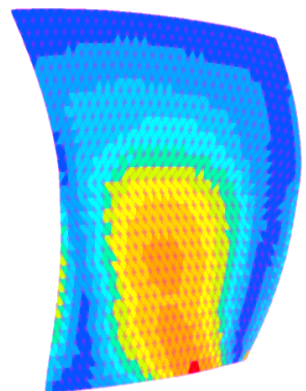

(a) $i t_{0}$

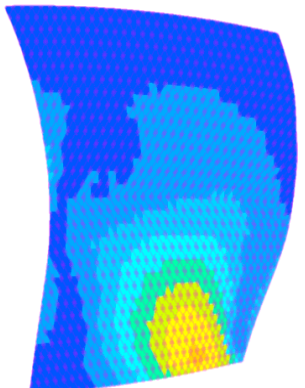

(b) $i t_{1}$

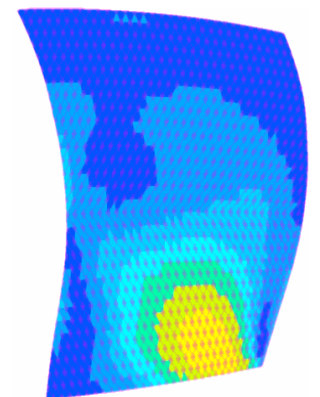

(c) $i t_{2}$

(d) color scale

Figure 3. Von mises stress fields within the blade (deformed geometry) at $\Omega^{*}$ from the suction side

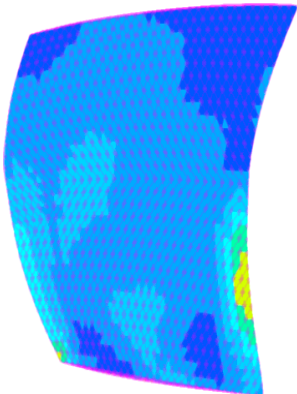

(a) $i t_{0}$

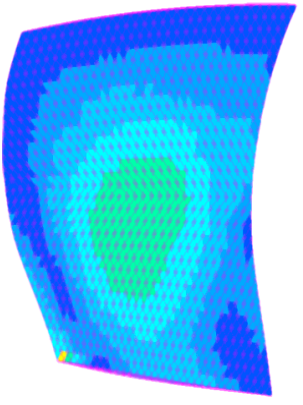

(b) $i t_{1}$

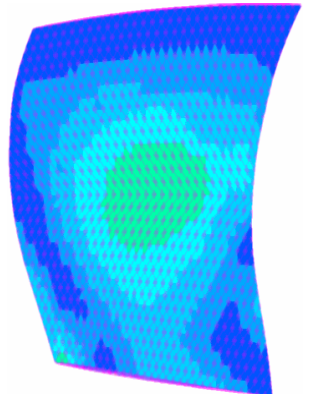

(c) $i t_{2}$

(d) color scale

Figure 4. Von mises stress fields within the blade (deformed geometry) at $\Omega^{*}$ from the pressure side

been carried out in order to match experimental observations. 


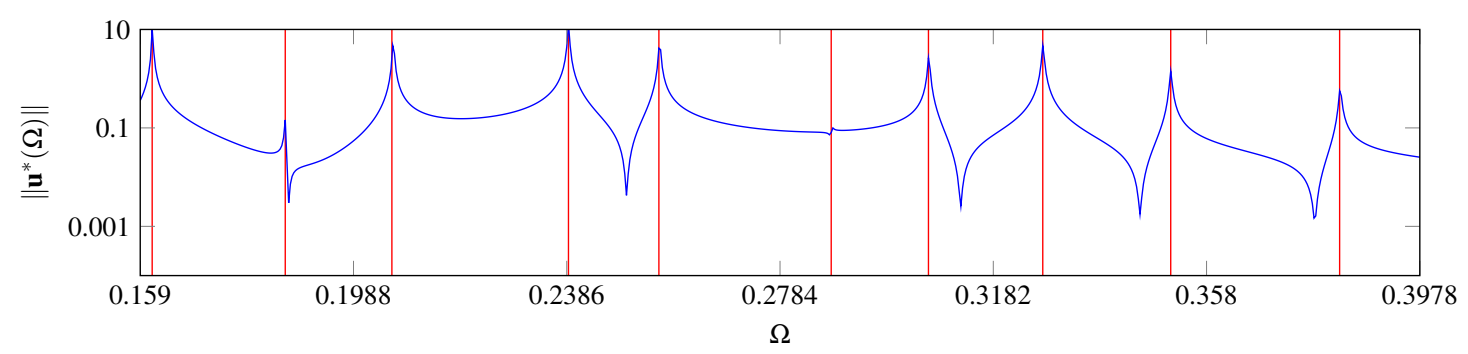

(a) profile $i t_{0}$

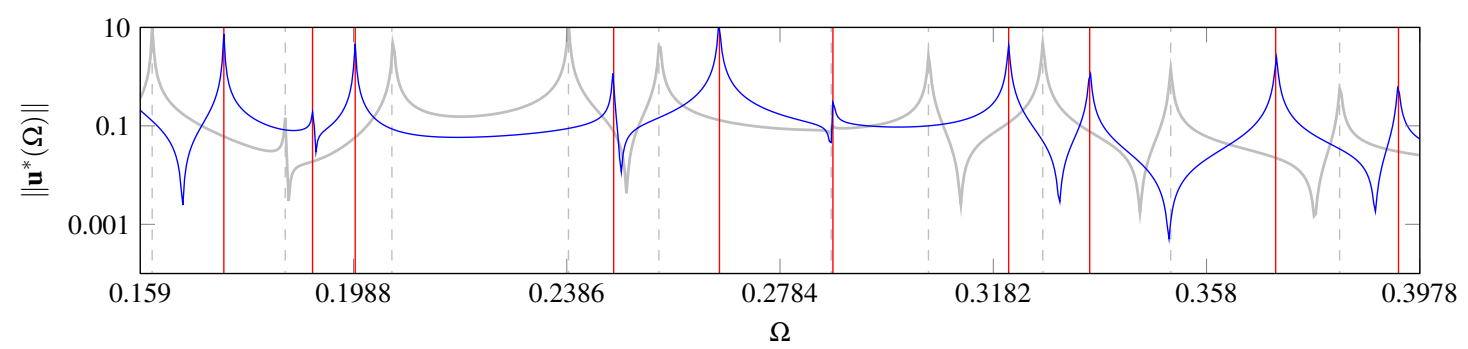

(b) profile $i t_{1}$

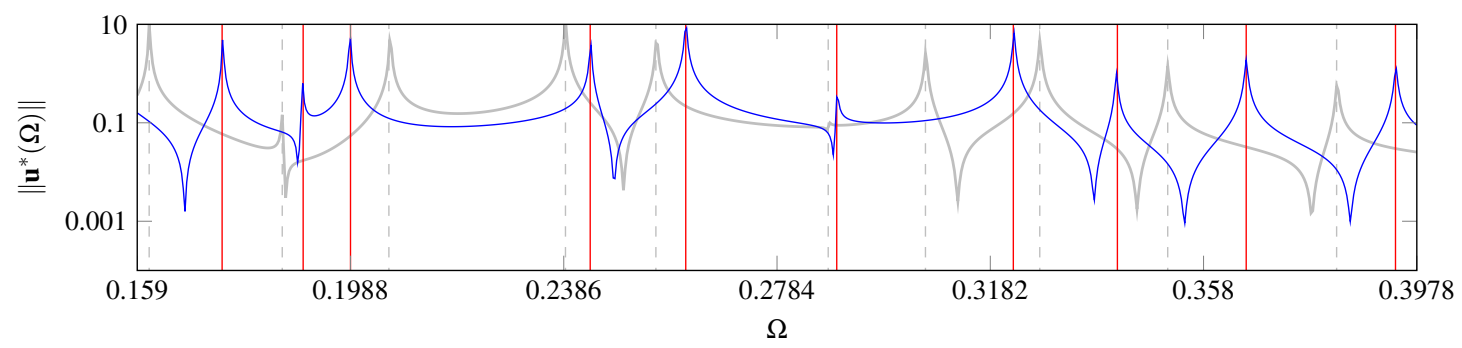

(c) profile $i t_{2}$

Figure 5. Linear forced response $((-))$ of the axial displacement under aerodynamic equivalent loading and comparison with the initial profile $((-))$.

\section{Optimization procedure}

The results obtained with 2 -lobe configuration contact simulations have been used in order to characterize key quantities associated with the blade vibratory behaviour. The exact definition of these criteria cannot be given for the sake of confidentiality. However, among the quantities of interest are the following:

1. the clearance consumption, meaning the incursion depth of the blade within the abradable coating;

2. the stress field in the blade during an interaction;

3. the behaviour of the vibration amplitudes for an interaction speed.

In agreement with experimental observations made on the rig test, extensive numerical simulations carried out in Snecma allowed for the identification of one particular critical rotational frequency denoted $\Omega^{*}$ in the following. The optimization criteria are used for the redesign of the blade profile: an iterated profile must allow for a very significant reduction of each of these three parameters. Optimization constraints feature standard mechanical considerations such as: (1) the mass of an iterated design may not be increased by more than $5 \%$ with respect to the initial design, (2) the static stress (which accounts for centrifugal stiffening, thermal and pressure loadings) cannot exceed a maximum value $\sigma_{\max }$ and (3) the eigenfrequencies of the iterated profiles must remain within a given range in order to avoid any modal interaction with upstream guidevanes or the surrounding casing.

An experimental design for the modification of the stacking law led to two iterated profiles, namely $i t_{1}$ and $i t_{2}$ that are compared in the following with the profile $i t_{0}$.

\section{Profiles comparison}

\subsection{Mechanical properties}

The three blade profiles are first compared with respect to their mass which are given in Tab. 1. The mass variation from a profile to another is quite small, up to $4.15 \%$ for the $i t_{2}$ profile, and is the consequence of the modification of the stacking law. There is a correlation between a blade mass and its stacking law. Indeed, in order to avoid dangerous vibratory behaviours after structural contacts occur, a well-known design strategy lies in facilitating clearance opening after contact which can be achieved with a more pronounced stacking law curvature. However, in order to limit subsequent static stresses increase, the blade profiles may be slightly enlarged thus leading to added mass.

\begin{tabular}{l|rrr}
\hline profile & $i t_{0}$ & $i t_{1}$ & $i t_{2}$ \\
mass evolution $(\%)$ & 0 & +1.45 & +4.15 \\
\hline
\end{tabular}

Table 1. Mass variation with respect to the initial profile

One of the main components of the static stress stems from centrifugal stiffening. The maximum value of this stress (using the Von Mises criterion) through the blade profile for the rotational frequency $\Omega^{*}$ is denoted $\sigma_{\mathrm{c}}$ in the following. Values obtained for each profile are given in Tab. 2. Both iterated profiles feature significant improvement in terms of centrifugal stresses since the maximum computed stress for blade profiles $i t_{1}$ and 


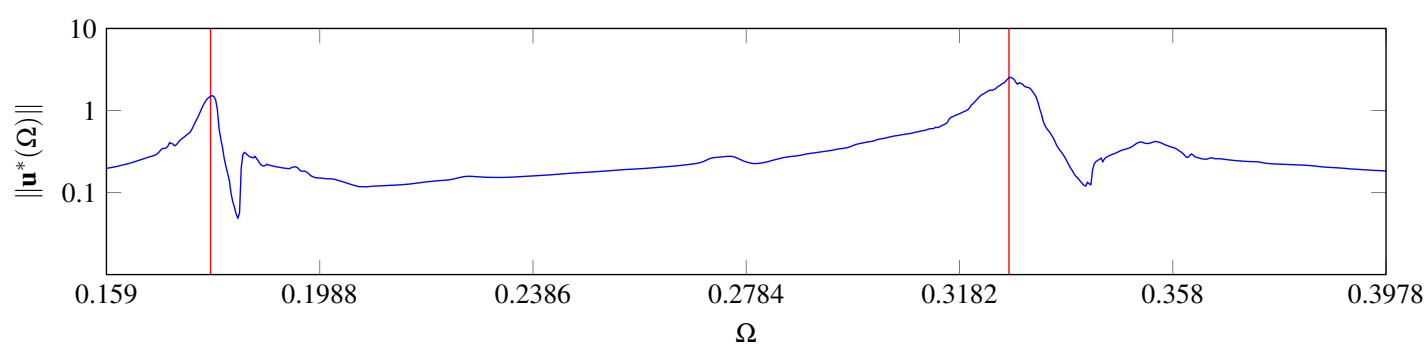

(a) profile $i t_{0}$

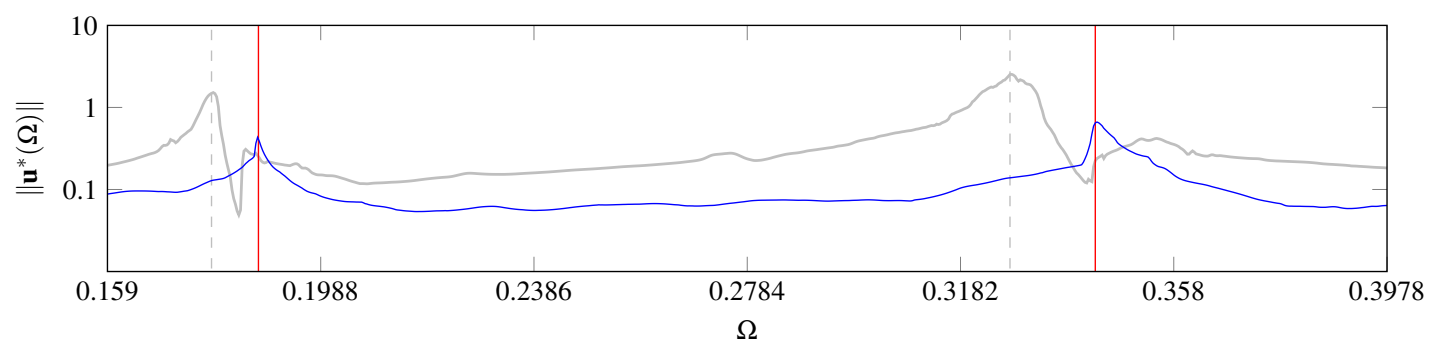

(b) profile $i t_{1}$

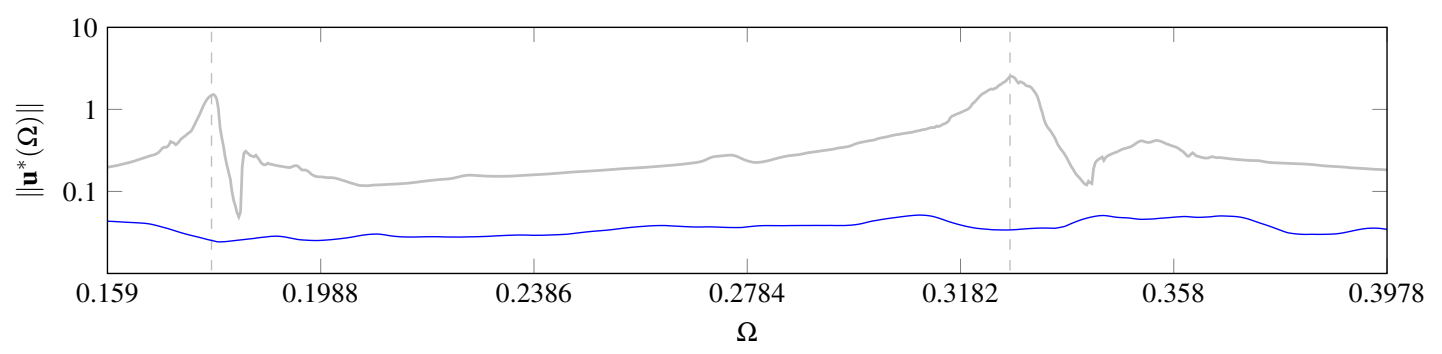

(c) profile $i t_{2}$

Figure 6. Amplitude $((-))$ of the leading edge axial displacement when structural contacts occur and comparison with the initial profile $((-))$.

$i t_{2}$ are respectively $28.32 \%$ and $31.90 \%$ lower than the one computed with the initial profile.

\begin{tabular}{l|rrr}
\hline profile & $i t_{0}$ & $i t_{1}$ & $i t_{2}$ \\
$\sigma_{c}$ evolution $(\%)$ & 0 & -28.32 & -31.90 \\
\hline
\end{tabular}

Table 2. EVOLUTION OF $\sigma_{c}$ WITHIN THE BLADE PROFILE

Full stress maps for the three considered blade profiles are depicted in Fig. 3 for the suction side and in Fig. 4 for the pressure side.

It is visible that compared to the initial profile $i t_{0}$, iterated profiles $i t_{1}$ and $i t_{2}$ feature much more localized high stress areas, particularly close to the blade root and the trailing edge. Interestingly, the area of high stresses visible on the trailing edge on the pressure side of the initial profile in Fig. 4 vanishes for both iterated profiles thus hinting a much lower risk of crack initiation in this area.

\begin{tabular}{l|rrr}
\hline profile & $i t_{0}$ & $i t_{1}$ & $i t_{2}$ \\
$f_{1 \mathrm{~B}}$ evolution (\%) & 0 & +5.76 & +3.44 \\
\hline$f_{1 \mathrm{~T}}$ evolution $(\%)$ & 0 & +1.93 & -2.10 \\
\hline
\end{tabular}

Table 3. Two first eigenfrequencies at rest

Finally, the three blade profiles are compared with respect to their two first eigenfrequencies at rest - the first bending mode $f_{1 \mathrm{~B}}$ and the first torsional mode $f_{1 \mathrm{~T}}$-in Tab. 3. As mentioned above, both frequencies must remain within a close range against the values of the initial profile in order to avoid potential modal interactions. Variations for both the first bending mode and the first torsional mode are acceptable with only a few percent variation.

\subsection{Linear forced response}

The airflow applies a pressure loading on the blade that is dependent on its angular position with respect to the upstream guide vanes. A weak coupling is assumed: CFD simulations are only used in order to define the applied sinusoidal pressure loading on the blade surface. The forced response to this pressure loading is computed over a properly selected rotational frequency range. Centrifugal stiffening is accounted for.

The forced response of each blade profile is pictured in Fig. 5, the amplitude of the blade leading edge axial displacement is shown for each rotational frequency once steady state has been reached. The forced response obtained for profile $i t_{0}$ is superimposed on Figs. 5(b) and 5(c) to ease the comparison between profiles. Throughout the rotational frequency range of interest, critical rotational frequencies-rotational frequencies for which a peak of displacement occurs — are marked in $((-))$.

Differences between the three profiles are not patent outside of the rotational frequency range $\Omega \in[0.1988 ; 0.2395]$ over which both iterated profiles feature signigicantly lower amplitudes of vibration. The modification of the first eigenfrequencies underlined in Tab. 3 logically implies that both iterated profiles have distinct critical velocities but the overall number of peaks is identical for each profile.

The similarity between the forced response of each profile is consistent with the fact that each profile features identical aerodynamic properties. 


\subsection{Contact simulations with 2 lobes}

Contact simulations are carried out over the same rotational frequency range considered in the previous section. For each rotational frequency, the amplitude of the leading edge axial displacement is retained once steady state has been reached. The evolution of this quantity is plotted for each blade profile in Fig. 6. Figures 6(b) and 6(c) feature both the results for an iterated profile and the initial profile to ease comparison. First of all, it is noticeable that two critical rotational frequencies are detected for the initial profile. Similarly to what was observed in [7] in another stage of an aircraft engine, these rotational frequencies feature increased amplitude of displacements as well as sophisticated abradable removal pattern on the casing but the precise analysis of such interaction goes beyond the scope of this study. One may notice that both iterated profiles feature much lower amplitudes of vibration, with almost one order of magnitude lower for the profile $i t_{2}$ in comparison with the initial profile thus confirming $a$ posteriori the relevance of the optimization criteria defined above. However, it is important to note that these results are not representative of the abradable coating removal on the casing or the maximum amplitude of vibration during the transient part of the interaction. Nevertheless, these results indicate a much smoother behaviour once steady state is reached and much lower stresses within the blade which is critical in order to ensure an acceptable service life. The shift between the critical rotational frequencies of profiles $i t_{0}$ and $i t_{1}$ is about $6 \%$ in agreement with the variation of the first eigenfrequency $f_{1 \mathrm{~B}}$ given in Tab. 3 . This confirms that both detected interactions are related to bending motions. In this context, the absence of critical rotational frequencies for the $i t_{2}$ profile is remarkable.

In order to better visualize the influence of the blade profile over the predicted abradable coating removal, the abradable coating profiles at the end of each simulation are depicted in Fig. 7 for each blade. For each rotational frequency, the level of abradable removal-meaning the wear pattern along the casing circumference-is depicted with a colour code from white (no abradable coating removal) to black (areas for which the abradable coating removal is maximum). The juxtaposition of these profiles over the rotational frequency range leads to the maps depicted in Figs. 7(a), 7(b) and 7(c). The two dominant black areas stem from the casing ovalization which creates two privileged contact areas along the circumference. In addition to the quantity of abradable coating removed, these maps provide useful insight regarding the transient vibratory response of the blade since any significant amplitude of vibration that leads to abradable removal would appear as a permanent dark area on the map no matter the amplitude of vibration once a steady state has been reached. Critical rotational frequencies $((-))$ detected in Fig. 6 are a match with the deepest wear patterns observed for each profile. In the case of the profile $i t_{0}$, one can see that four dark areas exist for $\Omega \simeq 0.32$ which illustrates that the blade impacts the casing four times per revolution. Interestingly, the maps show great consistency with the results depicted in Fig. 6 in the sense that no significant abradable removal is predicted far from the critical rotational frequencies. This indicates that transient motions do not feature elevated amplitudes with significant abradable removal. Thus the optimized profiles not only feature smaller amplitudes of vibration once a steady state has been reached, but numerical simulations also predict that they do not feature peaks of vibration during transients.

In regards of previous results obtained for different types of blades by the authors such as the profile modification study carried out for low-pressure compressor blades in [7], both results depicted in Fig. 6(c) and 7(c) are noteworthy. Indeed, while the shift of a critical rotational frequency from a profile to another has already been predicted with potentially lower levels of abradable removal, the very disappearing of these critical rotational speeds was unexpected. In order to assess the robustness of the optimized profiles, a second interaction case with four contact areas is considered in the following section.

\subsection{Contact simulations with 4 lobes}

Contact simulations now feature a casing deformed with four privileged contact areas as depicted in Fig. 2. It should be mentioned here that doubling the number of contact areas is not equivalent to a doubling of the rotational frequency with two contact areas due to the fact that centrifugal stiffening is accounted for. Same as in previous sections, Fig. 8 pictures the evolution of the blade leading edge axial displacement for each profile. For such a contact configuration, the $i t_{0}$ profile features a relatively high number of peaks with a dominant interaction frequency at $\Omega \simeq 0.331$. This interaction frequency is still visible for the $i t_{1}$ profile in Fig. 8(b), slightly shifted in agreement with Tab. 3 but all other peaks vanish. Blade profiles $i t_{1}$ and $i t_{2}$ both feature lower amplitude of vibration as well as a much smoother evolution of the amplitude over the rotational frequency range. Thus, obtained results are consistent with those for a 2-lobe contact configuration and show that the optimization criteria defined with respect to a 2-lobe configuration remain relevant for other interaction scenarios.

\section{Results analysis}

In order to better apprehend the fundamental differences between the initial profile and iterated profile $i t_{2}$, it is proposed to analyse the time signals obtained for $\Omega^{*}$ with the 2-lobe contact configuration. For each profile, the spectrogram of the blade leading edge axial displacement over time is pictured in Figs. 9(a) and 9(b).

Each of these spectrograms reveal the evolution of the frequency content of the blade response over time. The colour scale is normalized with respect to the maximum displacement for each configuration, from white to black where the highest amplitudes are reached. It is first visible that the spectrum of profile $i t_{0}$ is widely spread over the frequency domain, from 0 to 15 . To the contrary, the spectrum obtained for profile $i t_{2}$ is much more localized with a clearly dominant narrow band between 0 and 7.5.

These spectrograms emphasize the very distinct vibratory behaviours of the two profiles. When contact occurs, in the 2-lobe contact configuration considered in this paper, the blade profile $i t_{2}$ features a much more compact spectrogram that essentially involves the first free vibration modes. Thus the deformation of the blade when blade-tip/abradable coating contact occurs is fully captured by low frequency free vibration modes.

The optimization process mostly affects the upper half of the blade. This seems consistent with the fact that the added optimization criteria mostly stem from the blade structural behaviour at the vicinity of the blade tip.

\section{Conclusion}

This study focuses on the redesign of a high-pressure compressor blade for which structural divergences were witnessed after structural contacts had occurred. A previously introduced numerical strategy for the simulation of such interaction accounting for centrifugal stiffening and the abradable coating removal has 


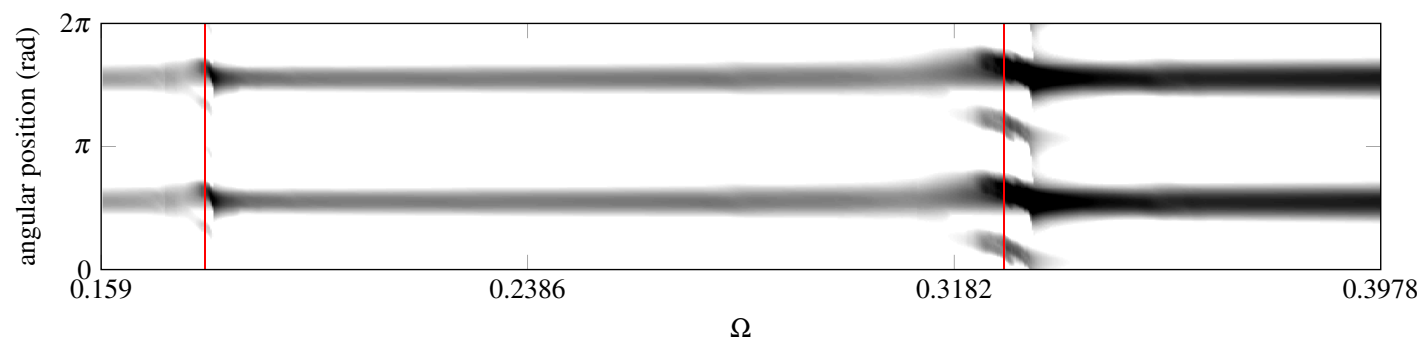

(a) profile $i t_{0}$

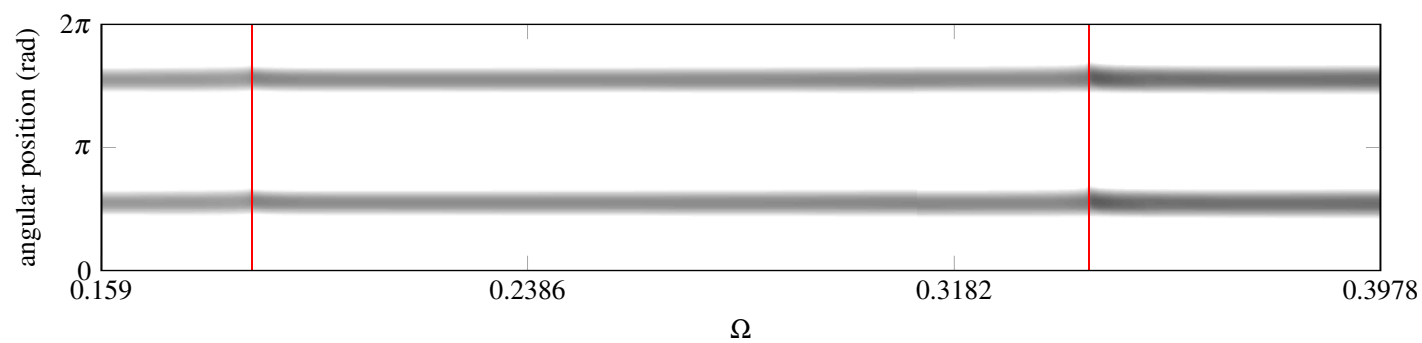

(b) profile $i t_{1}$

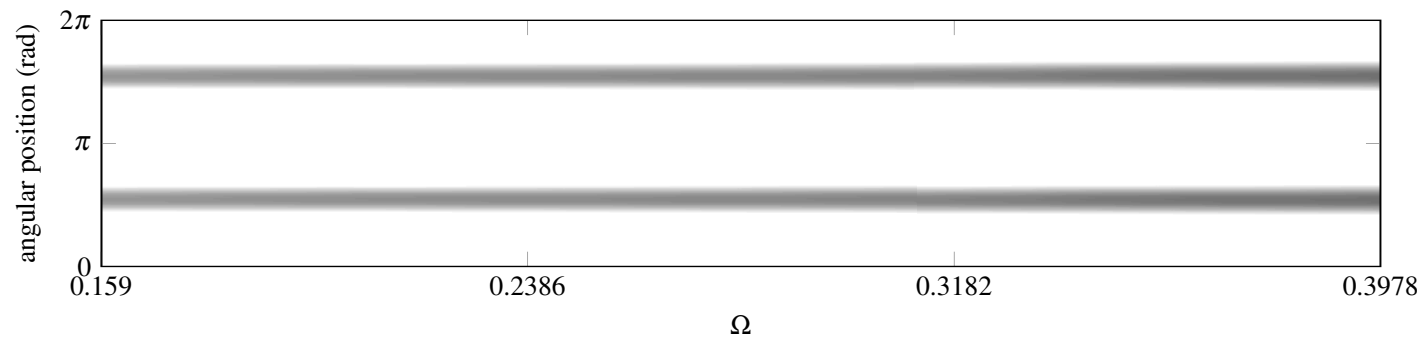

(c) profile $i t_{2}$

no removal

maximum removal

(d) color scale

Figure 7. Level of abradable removal along the casing circumference at the end of the simulations

been implemented within the industrial environment of Snecma. Based on the results of contact simulations, optimization criteria are defined for the blade of interest and accounted for in the redesign process. Two iterated profiles featuring identical aerodynamic properties to the initial profile are obtained and compared with respect to key mechanical parameters. It is first underlined that iterated profiles advantageously lead to a significant drop of the maximum static stress within the blade. Further simulations, forced response under aerodynamic loading and vibratory response to two distinct contact configurations over a properly selected rotational frequency range, confirm the fundamental improvements obtained with these iterated profiles. Work is in progress for expanding the work presented in this paper to a more general strategy of blade profile optimization with respect to structural contacts for different stages of an aircraft engine.

\section{Acknowledgement}

Thanks go to Turbomeca and Snecma for their technical and financial support.

\section{References}

[1] C. Padova, J. Barton, M. Dunn, S. Manwaring, Experimental results from controlled blade tip/shroud rubs at engine speed, J Turbomach 129 (4) (2007) 713-723, DOI:10.1115/1.2720869.
[2] R. Williams, Simulation of blade casing interaction phenomena in gas turbines resulting from heavy tip rubs using an implicit time marching method, in: Proceedings of the ASME Turbo Expo 2011-GT2011-45495, Vancouver, Canada, 2011, DOI:10.1115/GT2011-45495.

[3] P. Schmiechen, Travelling wave speed coincidence, Ph.D. thesis, College of Science, Technology and Medicine, London, UK (1997).

[4] A. Batailly, M. Legrand, P. Cartraud, C. Pierre, Assessment of reduced models for the detection of modal interaction through rotor stator contacts, Journal of Sound and Vibration 329 (2010) 5546-5562, DOI:10.1016/j.jsv.2010.07.018.

[5] D. Childs, A. Bhattacharya, Prediction of dry-friction whirl and whip between a rotor and a stator, Journal of Vibration and Acoustics 129 (2007) 355-362, DOI:10.1115/1.2731412.

[6] M. Legrand, A. Batailly, B. Magnain, P. Cartraud, C. Pierre, Full three-dimensional investigation of structural contact interactions in turbomachines, Journal of Sound and Vibration 331 (11) (2012) 2578-2601, DOI:10.1016/j.jsv.2012.01.017.

[7] A. Batailly, M. Legrand, A. Millecamps, G. F., Numericalexperimental comparison in the simulation of rotor/stator 


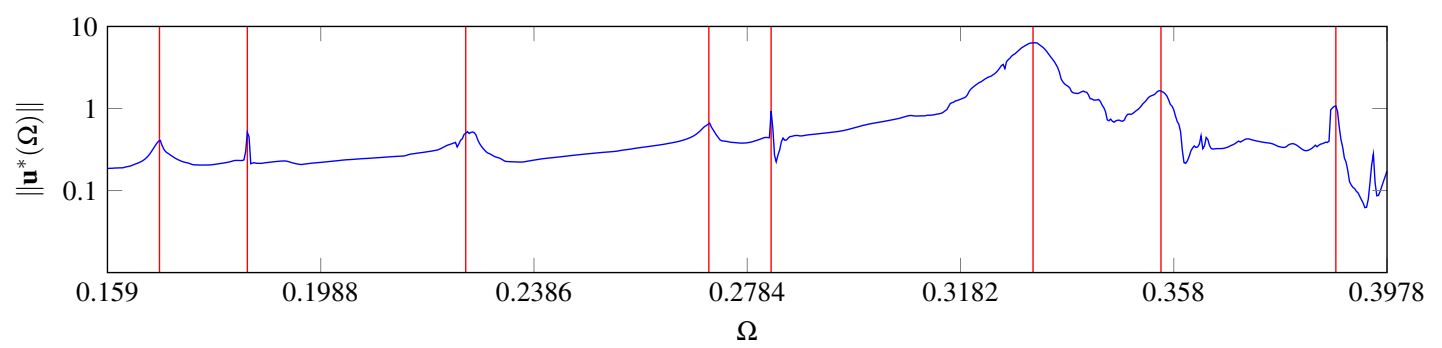

(a) profile $i t_{0}$

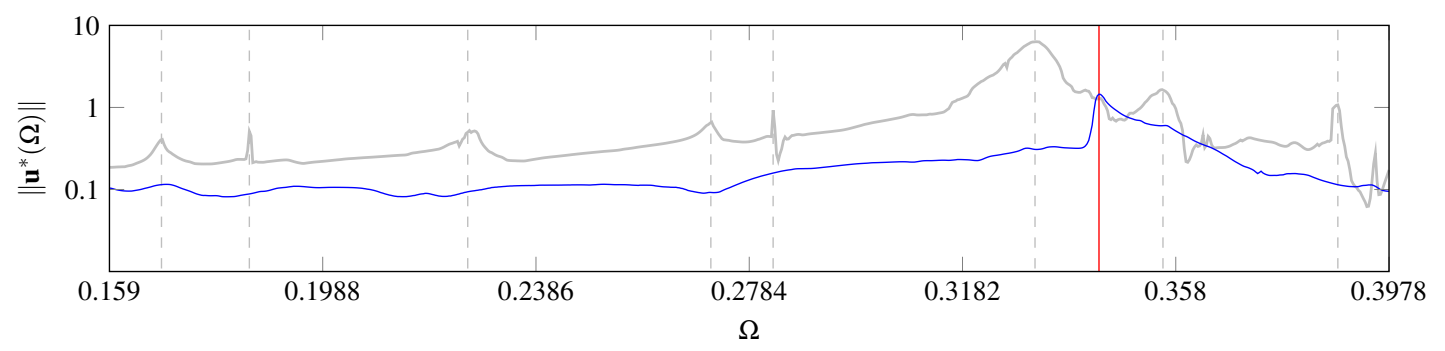

(b) profile $i t_{1}$

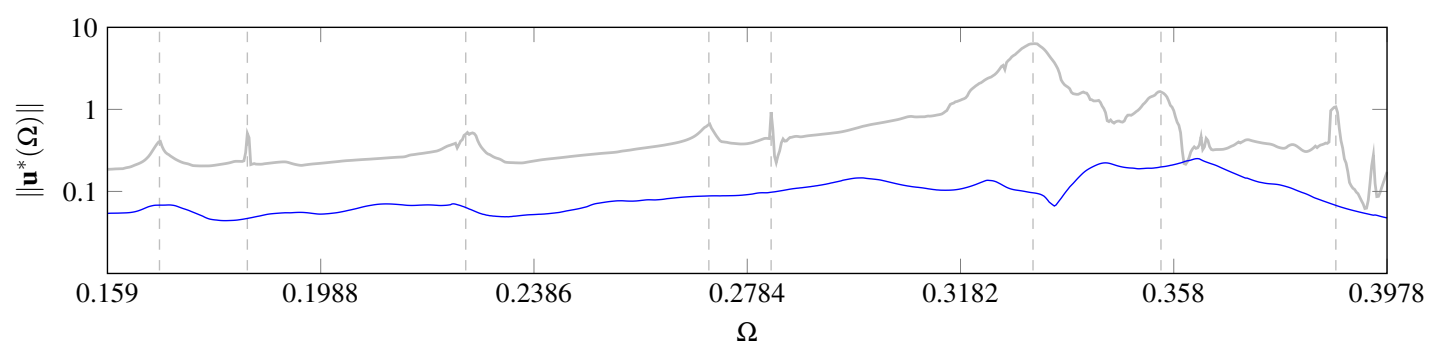

(c) profile $i t_{2}$

Figure 8. Amplitude $((-))$ of the leading edge axial displacement when structural contacts occur and comparison with the initial profile $((-))$.

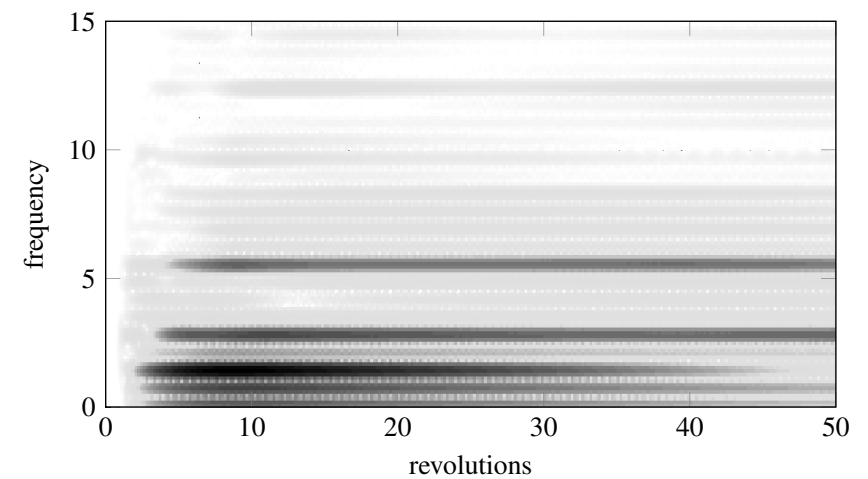

(a) profile $i t_{0}$

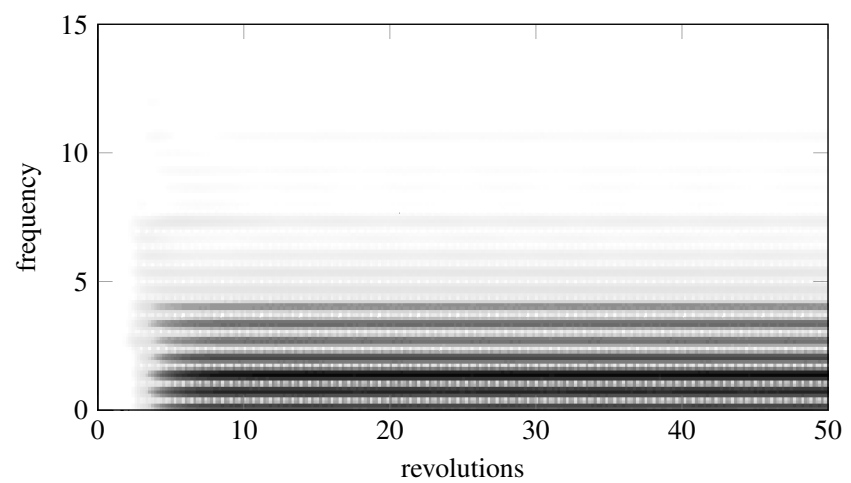

(b) profile $i t_{2}$

Figure 9. Spectrograms

interaction through blade-tip/abradable coating contact, J Eng Gas Turb Power 134, DOI:10.1115/1.4006446.

[8] A. Millecamps, J. Brunel, P. Dufrénoy, F. Garcin, M. Nucci, Influence of thermal effects during bladecasing contact experiments, in: Proceedings of the ASME IDETC/CIE-DETC2009-86842, San Diego, USA, 2009, DOI:10.1115/DETC2009-86842.

[9] N. Salvat, A. Batailly, M. Legrand, Modeling of abradable coating removal in aircraft engines through delay differential equations, J Eng Gas Turb Power 135 (10) (2013) 102102,
DOI:10.1115/1.4024959.

[10] V. Perrot, A. Pesteil, Turbine engine blade having improved stacking law (06 2012).

URL http://www.patentlens.net/patentlens/ patents.html?patnums=WO_2012_080669_A1\& language $=$ en\&query $=\% 28 W 02012080669 \_A 1 \%$ 20 in $\% 20$ publication_number $\% 29 \&$ stemming= true\&returnTo=structured.html $\% 3$ Fquery $\% 3 \mathrm{D} \%$ 2509W02012080669\%2BA1\#tab_1 